PROCEEDINGS OF THE

AMERICAN MATHEMATICAL SOCIETY

Volume 129, Number 1, Pages 165-172

S 0002-9939(00)05541-6

Article electronically published on June 14, 2000

\title{
UNBOUNDED QUASI-INTEGRALS
}

\author{
ALF BIRGER RUSTAD
}

(Communicated by Palle E. T. Jorgensen)

\begin{abstract}
Let $X$ be a locally compact Hausdorff space. We define a quasimeasure in $X$, a quasi-integral on $C_{0}(X)$, and a quasi-integral on $C_{c}(X)$. We show that all quasi-integrals on $C_{0}(X)$ are bounded, continuity properties of the quasi-integral on $C_{c}(X)$, representation of quasi-integrals on $C_{c}(X)$ in terms of quasi-measures, and unique extension of quasi-integrals on $C_{c}(X)$ to $C_{0}(X)$.
\end{abstract}

\section{INTRODUCTION}

The notion of a quasi-measure was introduced in 1 1 by J. F Aarnes. In [1, physical states on commutative unital $\mathrm{C}^{*}$-algebras were represented by quasi-measures. The quasi-measure in [1] was defined as a regular, finitely additive set function on open and closed subsets of a compact Hausdorff space $X$. The quasi-integral (physical state) with respect to a quasi-measure was constructed on the space of continuous functions on $X$ (denoted $C(X)$ ). The quasi-integrals were shown to be the maps linear on each uniformly closed, singly generated subalgebra of $C(X)$.

Recent results (cf. [2], 4] and 6]) indicate that the quasi-measures are interesting as a generalization of regular Borel measures. The restriction of a quasi-measure to a compact Hausdorff space is therefore unfortunate. Accordingly, the work presented here aims to extend the theory in [1] to $X$ being a locally compact Hausdorff space.

In the sequel we let $X$ denote a locally compact Hausdorff space. A set is called bounded if its closure is compact. $\mathcal{F}$ and $\mathcal{O}$ denote respectively the class of closed and the class of open subsets of $X$. Similarly, $\mathcal{C}$ and $\mathcal{O}^{*}$ denote respectively the class of compact and the class of open bounded subsets. Furthermore we put $\mathcal{A}=\mathcal{F} \cup \mathcal{O}$ and $\mathcal{A}^{*}=\mathcal{C} \cup \mathcal{O}^{*}$.

Definition 1.1. A quasi-measure in $X$ is a function $\mu: A \rightarrow[0, \infty]$ satisfying the following conditions:

1. $\mu(A)<\infty$ if $A \in \mathcal{A}^{*}$.

2. For any finite, disjoint collection $\left\{A_{i}\right\}_{i=1}^{n} \subset \mathcal{C} \cup \mathcal{O}$ with $\bigcup_{i=1}^{n} A_{i} \in \mathcal{C} \cup \mathcal{O}$, then

$$
\mu\left(\bigcup_{i=1}^{n} A_{i}\right)=\sum_{i=1}^{n} \mu\left(A_{i}\right) .
$$

3. $\mu(U)=\sup \{\mu(K): K \subset U, K \in \mathcal{C}\}, U \in \mathcal{O}$.

4. $\mu(F)=\inf \{\mu(U): F \subset U, U \in \mathcal{O}\}, F \in \mathcal{F}$.

Received by the editors October 14, 1996 and, in revised form, March 22, 1999

1991 Mathematics Subject Classification. Primary 28A25. 
Our quasi-measure corresponds to the quasi-measure in [3], and the reader will find numerous properties of the quasi-measure there. The notion of a quasi-measure in a locally compact Hausdorff space can also be found in [7]. The definition in [7] is more restrictive than ours and does not produce the quasi-integrals given below.

Let $C_{0}(X)$ denote the real-valued continuous functions on $X$ vanishing at infinity and let $C_{c}(X)$ be the functions in $C_{0}(X)$ with compact support. The support of a function $a \in C_{0}(X)$ will be denoted by supp $a$ and the range of $a$ in $\mathbf{R}$ by $\operatorname{sp} a$. If $a \in C_{0}(X)$, let $\mathbf{A}_{0}(a)$ denote the smallest uniformly closed subalgebra of $C_{0}(X)$ containing $a$. For subsets $K, O \subset X$ we will let $K \prec a$ and $a \prec O$ denote that $a \in C_{c}(X), \operatorname{sp} a \subset[0,1]$ and respectively that $x \in K \Rightarrow a(x)=1$ and supp $a \subset O$.

Definition 1.2. A real-valued function $\rho$ on $C_{0}(X)$ is called a quasi-integral if the following conditions are satisfied:

1. $b \geq 0 \Rightarrow \rho(b) \geq 0$ whenever $b \in C_{0}(X)$.

2. $\rho$ is linear on $\mathbf{A}_{0}(a)$ for each $a \in C_{0}(X)$.

When $\sup \{\rho(a): a \prec X\}<\infty$ we say that $\rho$ is bounded. If in addition $\sup \{\rho(a)$ : $a \prec X\}=1$ we say that $\rho$ is a quasi-state.

In the $\mathrm{C}^{*}$-algebra setting this corresponds to the commutative, nonunital case, where $\rho$ is characterized by linearity on closed subalgebras generated by self-adjoint elements.

If $a \in C_{c}(X)$, then we have $\mathbf{A}_{0}(a) \subset C_{c}(X)$. Hence we may define a quasiintegral on $C_{c}(X)$ similarly as above:

Definition 1.3. A real-valued function $\rho$ on $C_{c}(X)$ is called a quasi-integral if:

1. $f \geq 0 \Rightarrow \rho(f) \geq 0$ whenever $f \in C_{c}(X)$.

2. $\rho$ is linear on $\mathbf{A}_{0}(f)$ for each $f \in C_{c}(X)$.

If in addition $\sup \{\rho(f): f \prec X\}<\infty$, then $\rho$ is bounded and we put $\|\rho\|=$ $\sup \{\rho(f): f \prec X\}$.

The only difference between the definition above and Definition 1.2 is that $\rho$ is now restricted to $C_{c}(X)$. However we will show that if $\rho$ is bounded, these two definitions coincide (Corollary 3.10). The key results in this article are boundedness of quasi-integrals on $C_{0}(X)$ and a representation theorem between the quasi-measures in $X$ and the quasi-integrals on $C_{c}(X)$. The representation is a generalization of the Riesz Representation Theorem in [5].

The section below presents some preparatory results on the quasi-measures and quasi-integrals on $C_{0}(X)$. The section ends with the boundedness theorem for quasi-integrals on $C_{0}(X)$. The next and last section presents construction of the quasi-integral on $C_{c}(X)$ with respect to a quasi-measure. Monotonicity and continuity properties of the quasi-integral are given. The section highlights with the representation theorem for quasi-measures and quasi-integrals on $C_{c}(X)$. Finally, unique extension to $C_{0}(X)$ of quasi-integrals on $C_{c}(X)$ is given.

\section{Quasi-Integrals on $C_{0}(X)$}

Throughout this article we will assume that $X$ is a locally compact Hausdorff space. The results in the following proposition were given in [3]. We will only give a brief outline of the proofs here. 
Proposition 2.1. Let $\mu$ be a quasi-measure in $X$.

1. $\mu(\emptyset)=0$.

2. $A \subset B \Rightarrow \mu(A) \leq \mu(B), A, B \in \mathcal{A}$.

3. If $K \in \mathcal{C}, F \in \mathcal{F}$ are disjoint, then $\mu(F \cup K)=\mu(F)+\mu(K)$.

4. $\mu$ is countably additive on open sets.

5. Let $\mu$ be a quasi-measure in $X$. For any increasing family of open sets $\left\{V_{\lambda}\right\}$, if $V_{\lambda} \nearrow V$ (i.e. $\left.\bigcup V_{\lambda}=V\right)$, then $\mu\left(V_{\lambda}\right) \nearrow \mu(V)$.

Proof. With $A_{1}=A_{2}=\emptyset$ in item 2 of Definition 1.1 we get $\mu(\emptyset)=0$. The monotonicity follows from regularity (items 3 and 4 of Definition [1.1). The third statement follows from regularity and a Urysohn's lemma argument. The fifth statement follows from regularity (item 3 of Definition 1.1). The fifth statement and finite additivity (item 2 of Definition 1.1) imply the fourth statement.

Proposition 2.2. A set function $\mu: A \rightarrow[0, \infty]$ satisfying items 1,3 , and 4 of Definition 1.1 is a quasi-measure if and only if the following are satisfied:

1. If $O_{1}, O_{2} \in O$ are disjoint, then $\mu\left(O_{1} \cup O_{2}\right)=\mu\left(O_{1}\right)+\mu\left(O_{2}\right)$.

2. If $K \subset O \in O$ with $K$ compact, then $\mu(O)=\mu(O \backslash K)+\mu(K)$.

Proof. The proof of the third statement in Proposition 2.1 holds for $\mu$. Hence by induction $\mu$ is finitely additive on $\mathcal{C}$. Similarly, $\mu$ is finitely additive on $\mathcal{O}$ by assumption 2.2. 1. Let $\left\{A_{i}\right\}_{i=1}^{n} \subset \mathcal{C} \cup \mathcal{O}$ with disjoint union $A=\bigcup_{i=1}^{n} A_{i} \in \mathcal{C} \cup \mathcal{O}$. We may split the union to a disjoint union of a compact and an open set by $A=\left(\bigcup_{A_{i} \in \mathcal{C}} A_{i}\right) \cup\left(\bigcup_{A_{i} \notin \mathcal{C}} A_{i}\right)$. If $A$ is open, then $\mu(A)=\mu\left(\bigcup_{A_{i} \in \mathcal{C}} A_{i}\right)+\mu\left(\bigcup_{A_{i} \notin \mathcal{C}} A_{i}\right)$ by assumption 2.22 . With $\mu$ finitely additive on $\mathcal{C}$ and on $\mathcal{O}$ we obtain $\mu\left(\bigcup_{i=1}^{n} A_{i}\right)=$ $\sum_{i=1}^{n} \mu\left(A_{i}\right)$. If $A$ is compact we may use a similar argument. Hence it suffices to show that if $O$ is open and $O \subset K \in \mathcal{C}$, then $\mu(K)=\mu(K \backslash O)+\mu(O)$. Let $K^{\prime} \subset O$ be compact. Then since $\mu$ is monotone $\mu(K) \geq \mu(K \backslash O)+\mu\left(K^{\prime}\right)$. Taking supremum of all $K^{\prime} \subset O$, regularity yields $\mu(K) \geq \mu(K \backslash O)+\mu(O)$. Conversely, given $\epsilon>0$ pick an open set $U \supset K \backslash O$ with $\mu(U)<\mu(K \backslash O)+\epsilon$. Observing that $K \backslash U \subset O$ yields

$$
\begin{aligned}
\mu(K) & \leq \mu(U \cup O)=\mu(U)+\mu(K \backslash U) \\
& <\mu(K \backslash O)+\mu(O)+\epsilon .
\end{aligned}
$$

Equality follows. We have shown finite additivity for $\mu$ on $\mathcal{C} \cup \mathcal{O}$ which completes the proof.

Lemma 2.3. A quasi-integral $\rho$ on $C_{0}(X)$ is bounded on $\mathbf{A}_{0}(a)$ for each $a \in$ $C_{0}(X)$.

Proof. Suppose $\sup \left\{\rho(f): 0 \leq f \leq 1, f \in \mathbf{A}_{0}(a)\right\}=\infty$ for some $a \in C_{0}(X)$. Choose $\phi_{i}$ with $\phi_{i} \circ a \in \mathbf{A}_{0}(a), \rho\left(\phi_{i}(a)\right)>2^{2 i}$ and $0 \leq \phi_{i}(a) \leq 1$ for $i=1,2, \ldots$. Then with $\phi=\sum_{i=1}^{\infty} 2^{-i} \phi_{i}$ we have $\phi \circ a \in \mathbf{A}_{0}(a), 0 \leq \phi(a) \leq 1$ and $\rho(\phi(a))=\infty$, which is a contradiction. Hence we must have $\rho$ bounded on $\mathbf{A}_{0}(a)$.

Remark 1. Note that $\rho$ is a linear functional on $\mathbf{A}_{0}(a)$ and thus boundedness implies that $\rho$ is continuous on $\mathbf{A}_{0}(a)$ for each $a \in C_{0}(X)$. Hence

$$
\sup \{\rho(a): a \prec X\}=\sup \left\{\rho(a): 0 \leq a \leq 1, a \in C_{0}(X)\right\}
$$

for all quasi-integrals $\rho$ on $C_{0}(X)$. Moreover, the complexification of $\mathbf{A}_{0}(a)$ is a $\mathrm{C}^{*}$-algebra so Lemma 2.3 is not a new result. We included it for completeness and the reader's convenience. 
Lemma 2.4. Suppose $a \in C_{c}(X)$ with $0 \leq a \leq 1$. Then there is a function $f \in$ $C_{c}(X)$ with supp $a \prec f$. Moreover, $\operatorname{supp} a \prec f \prec X$ implies that $a, f \in \mathbf{A}_{0}(a+f)$ and $\rho(a) \leq \rho(f)$.

Proof. If $a \in C_{c}(X)$, then $\operatorname{supp} a=K$ is compact. There is an open bounded set $V$ containing $K$. By Urysohn's lemma there is a function $f$ with $K \prec f \prec V$ which implies that $f \in C_{c}(X)$. Define $\phi_{1}$ and $\phi_{2}$ by

$$
\phi_{1}(x)=\left\{\begin{array}{cc}
1, & x \geq 1, \\
x, & x<1,
\end{array} \text { and } \phi_{2}=\left\{\begin{array}{cc}
x-1, & x \geq 1 \\
0, & x<1
\end{array}\right.\right.
$$

Then $\phi_{1}(a+f)=f$ and $\phi_{2}(a+f)=a$; thus $a, f \in \mathbf{A}_{0}(a+f)$ and we get $\rho(a) \leq \rho(f)$.

Theorem 2.5 (Boundedness of quasi-integrals). All quasi-integrals on $C_{0}(X)$ are bounded.

Proof. Let $\rho$ be a quasi-integral on $C_{0}(X)$ and $\operatorname{suppose} \sup \{\rho(a): a \prec X\}=\infty$. By Lemma 2.4 recursively construct a sequence $\left\{a_{i}\right\}_{i=1}^{\infty}$ where $\rho\left(a_{i}\right) \geq 2^{2 i}$ and $\operatorname{supp} a_{i} \prec a_{i+1} \prec X$ for each $i$. Let $f=\sum_{i=1}^{\infty} 2^{-i} a_{i}$. Then $f \in C_{0}(X)$ since $C_{0}(X)$ is complete. Define $\phi_{i}$ for $i=1,2, \ldots$ by

$$
\phi_{i}(x)=\left\{\begin{array}{cl}
1, & x \geq 2^{-i+1} \\
2^{i}\left(x-2^{-i}\right), & 2^{-i} \leq x \leq 2^{-i+1} \\
0, & x \leq 2^{-i}
\end{array}\right.
$$

we have $\phi_{i} \in C(\operatorname{sp} f), \phi_{i}(0)=0$ and $\phi_{i}(f)=a_{i}$ for each $i$ and thus $\left\{a_{i}\right\}_{i=1}^{\infty} \subset \mathbf{A}_{0}(f)$. Finally $f \geq 2^{-i} a_{i}$ implies $\rho(f) \geq 2^{-i} \rho\left(a_{i}\right) \geq 2^{i}$ for $i=1,2, \ldots$ which in turn implies that $\rho(f)=\infty$. This is a contradiction since $\rho$ is supposed to be a quasi-integral on $C_{0}(X)$; we may conclude that $\sup \{\rho(a): a \prec X\}<\infty$ so $\rho$ is bounded.

Remark 2. Theorem 2.5 shows that the local linearity of the quasi-integrals impose strong restrictions on their global behavior. This suggests that unbounded quasiintegrals on $C_{c}(X)$ may exhibit nice properties. Indeed, this is what we will devote the next and last section to.

\section{Quasi-Integrals ON $C_{c}(X)$}

Proposition 3.1. Suppose that $\mu$ is a quasi-measure in $X$ and $f \in C_{c}(X)$. Then there is a unique bounded regular Borel measure $\mu_{f}$ on $\mathbf{R} \backslash\{\mathbf{0}\}$ with $\mu_{f}(O)=$ $\mu\left(f^{-1}(O)\right)$ for all open sets $O \subset \mathbf{R} \backslash\{\mathbf{0}\}$.

Proof. Let $\check{f}(x)=\mu\left(f^{-1}(-\infty, x) \backslash\{0\}\right)$ which implies that $\check{f}$ is increasing. Since $f \in C_{c}(X)$ we have that $f(\operatorname{supp} f)$ is compact. Hence $\check{f}$ is constant outside an interval $[a, b]$ for some $a, b \in \mathbf{R}$. By Proposition $2.1 \check{f}\left(x^{-}\right)=\check{f}(x)$ for each $x \in \mathbf{R}$, so $\check{f}$ is continuous from the left. Thus $\mu_{f}[x, y)=\check{f}(y)-\check{f}(x)$ uniquely determines a regular Borel measure in $\mathbf{R}$ and by regularity $\mu_{f}(x, y)=\check{f}(y)-\check{f}\left(x^{+}\right)$. If $x_{\lambda} \in(x, y)$, then $\check{f}(y) \geq \mu\left(f^{-1}\left[\left(x_{\lambda}, y\right) \backslash\{0\}\right]\right)+\check{f}\left(x_{\lambda}\right)$ since $\mu$ is monotone; hence $\check{f}(y)-\check{f}\left(x^{+}\right) \geq$ $\mu\left(f^{-1}[(x, y) \backslash\{0\}]\right)$. Conversely finite additivity and monotonicity of $\mu$ yields

$$
\begin{aligned}
\check{f}(y) & =\mu\left(f^{-1}[(x, y) \backslash\{0\}]\right)+\mu f^{-1}(\{x\} \backslash\{0\})+\check{f}(x) \\
& \leq \mu\left(f^{-1}[(x, y) \backslash\{0\}]\right)+\check{f}\left(x_{\lambda}\right),
\end{aligned}
$$

so $\check{f}(y)-\check{f}\left(x^{+}\right) \leq \mu\left(f^{-1}[(x, y) \backslash\{0\}]\right)$. We have $\mu\left(f^{-1}[(x, y) \backslash\{0\}]\right)=\check{f}(y)-\check{f}\left(x^{+}\right)=$ $\mu_{f}(x, y)$, and since both $\mu$ and $\mu_{f}$ are countably additive on open sets, the proof is complete. 
Remark 3. We will call $\mu_{f}$ the measure corresponding to $\mu$ and $f$. Notice that Proposition 3.1 is stated only for open sets not containing $\{0\}$, whereas the proof produces a measure on $\mathbf{R}$ with $\mu_{f}(\{0\})=0$. This is convenient when the quasimeasure is an extended real-valued function. In fact, Lemma 3.3 is not valid unless zero is omitted.

Definition 3.2. A map $f \mapsto \mu_{f}$ from $C_{c}(X)$ into the regular Borel measures in $\mathbf{R} \backslash\{\mathbf{0}\}$ is consistent if $\mu_{\phi \circ f}=\mu_{f} \circ \phi^{-1}$ for each $f \in C_{c}(X)$ and $\phi \in C(\operatorname{sp} f), \phi(0)=$ 0 .

Lemma 3.3. Let $\mu$ be a quasi-measure in $X$. Let $\mu_{f}$ denote the measure corresponding to $\mu$ and $f \in C_{c}(X)$. Then the map $f \mapsto \mu_{f}$ is consistent.

Proof. Let $f \in C_{c}(X), \phi \circ f \in \mathbf{A}_{0}(f)$ and $K \subset \mathbf{R} \backslash\{0\}$ be compact. Now $0 \notin \phi^{-1}(K)$ implies:

$$
\begin{aligned}
\mu_{\phi \circ f}(K) & =\mu\left((\phi \circ f)^{-1}(K)\right) \\
& =\mu\left(f^{-1}\left(\phi^{-1}(K)\right)\right) \\
& =\mu_{f}\left(\phi^{-1}(K)\right) .
\end{aligned}
$$

Note that since $K$ is compact in $\mathbf{R} \backslash\{0\}$, then $K$ is compact in $\mathbf{R}$ by the identity map. So $f^{-1}\left(\phi^{-1}(K)\right)$ is a closed subset of $\operatorname{supp} f$, and thus compact. The result now follows from the regularity of $\mu_{f}$.

In the sequel we will assume that the measure corresponding to a quasi-measure $\mu$ and a function $f \in C_{c}(X)$ is extended to $\mathbf{R}$ by $\mu_{f}\{0\}=0$.

Proposition 3.4. Let $\rho$ be a quasi-integral on $C_{c}(X)$. If $f, g \in C_{c}(X)$ and $f \leq g$, then $\rho(f) \leq \rho(g)$.

Proof. Given $\delta>0$, suppose $f \geq 0$ and $g(x) \geq \delta+f(x)$ when $x \in \operatorname{supp} f$. Pick a natural number $n$ such that $n \delta>\max g$ and define $\phi_{i} \in C(\operatorname{sp} f), 1 \leq i \leq n$ by:

$$
\phi_{i}(x)= \begin{cases}0, & x \leq(i-1) \delta, \\ x-(i-1), & (i-1) \delta<x<i \delta, \\ \delta, & x \geq i \delta .\end{cases}
$$

Then $x \in \operatorname{supp} \phi_{i}(f) \Rightarrow \phi_{i}(g(x))=\delta$; thus $\phi_{i}(f), \phi_{i}(g) \in \mathbf{A}_{0}\left(\phi_{i}(f)+\phi_{i}(g)\right)$ which imply $\rho\left(\phi_{i}(f)\right) \leq \rho\left(\phi_{i}(g)\right)$. Now $\sum \phi_{i}(f)=f, \sum \phi_{i}(g)=g$ implies $\rho(f) \leq \rho(g)$. Given $\epsilon>0$, suppose now that $0 \leq f \leq g$, choose $h$ and $\delta>0$ with $\operatorname{supp} g \prec h \in$ $C_{c}(X)$ and $\rho(\delta h)<\epsilon$. We have $\rho(f) \leq \rho(g+\delta h)<\rho(g)+\epsilon$. Let $f \leq g \in C_{c}(X)$ be arbitrary. Then $f^{+}, f^{-} \in A(f)$ and $f^{+} \leq g^{+}, f^{-} \geq g^{-}$. We have $\rho(f)=$ $\rho\left(f^{+}\right)-\rho\left(f^{-}\right) \leq \rho\left(g^{+}\right)-\rho\left(g^{-}\right)=\rho(g)$ by the previous argument. The proof is complete.

Corollary 3.5. Let $\rho$ be a quasi-integral on $C_{c}(X)$ and let $K$ be an arbitrary compact subset of $X$. Then there is a $k \in \mathbf{R}$ such that whenever $\operatorname{supp} f_{i} \subset K, f_{i} \in$ $C_{c}(X)$ for $i=1,2$, we have:

$$
\left|\rho\left(f_{1}\right)-\rho\left(f_{2}\right)\right| \leq k\left\|f_{1}-f_{2}\right\| .
$$

Proof. Pick a $g \succ K$ and let $\rho(g)=k$. Then $f_{1} \leq f_{2}+g\left\|f_{1}-f_{2}\right\|$ which implies that $\rho\left(f_{1}\right)-\rho\left(f_{2}\right) \leq \rho(g)\left\|f_{1}-f_{2}\right\|$ and conversely $\rho\left(f_{2}\right)-\rho\left(f_{1}\right) \leq \rho(g)\left\|f_{1}-f_{2}\right\|$. But then we must have $\left|\rho\left(f_{1}\right)-\rho\left(f_{2}\right)\right| \leq k\left\|f_{1}-f_{2}\right\|$. 
Remark 4. In general $\rho$ is not uniformly continuous (since it is a generalization of regular Borel measures). However, $\rho$ is continuous with respect to the topology of uniform convergence on compacta. Hence this is a sharp result; we cannot expect stronger continuity properties.

Corollary 3.6. Let $\rho$ be a bounded quasi-integral on $C_{c}(X)$. Then for each pair $f_{1}, f_{2} \in C_{c}(X)$ we have

$$
\left|\rho\left(f_{1}\right)-\rho\left(f_{2}\right)\right| \leq\|\rho\|\left\|f_{1}-f_{2}\right\| .
$$

Proof. Pick a function $g \succ \operatorname{supp} f_{1} \cup \operatorname{supp} f_{2}$. Then $\rho(g) \leq\|\rho\|$ and the result follows from Corollary 3.5

Proposition 3.7. Let $\mu$ be a quasi-measure in $X$. Define

$$
\rho(f)=\int i d \mu_{f} \text { for each } f \in C_{c}(X),
$$

where $\mu_{f}$ is the measure corresponding to $\mu$ and $f$ and $i$ is the identity map on $\boldsymbol{R}$. Then $\rho$ is a quasi-integral on $C_{c}(X)$.

Proof. By the transformation theorem for integrals and Lemma 3.3, the result follows.

Lemma 3.8. Let $\mu$ be a quasi-measure in $X$ and let $\rho$ be the corresponding quasiintegral. Then for each open set $O \subset X$ we have:

$$
\mu(O)=\sup \{\rho(f): f \prec O\} .
$$

Moreover, if $\mu(X)<\infty$, then $\rho$ is bounded and $\|\rho\|=\mu(X)$.

Proof. First suppose $\mu(O)<\infty$. Choose a compact set $K \subset O$ with $\mu(K)>$ $\mu(O)-\epsilon$, and a function $f$ with $K \prec f \prec O$. We have:

$$
\begin{aligned}
\rho(f) & =\int_{\mathrm{sp} f} i d \mu_{f}=\int_{\{1\}} d \mu_{f}+\int_{(0,1)} i d \mu_{f} \\
& \geq \int_{\{1\}} d \mu_{f}=\mu_{f}(\{1\})=\mu\left(f^{-1}\{1\}\right) \\
& \geq \mu(K) \text { since } K \subset f^{-1}\{1\} .
\end{aligned}
$$

On the other hand we have:

$$
\begin{aligned}
\rho(f) & \leq \int_{\mathrm{sp} f} d \mu_{f} & & (\operatorname{sp} f \subset[0,1]) \\
& =\mu_{f}(\operatorname{sp} f \backslash\{0\}) & & \\
& =\mu\left(f^{-1}(0, \infty)\right) & & \left(f^{-1}(0, \infty)=f^{-1}(\operatorname{sp} f \backslash\{0\})\right) \\
& \leq \mu(O) & & \left(f \prec O \Rightarrow f^{-1}(0, \infty) \subset O\right) .
\end{aligned}
$$

These together imply $\mu(O)=\sup \{\rho(f): f \prec O\}$. If $\mu(O)=\infty$, then there is, for every natural number $n$, a compact set $K \subset O$ with $\mu(K)>n$. By the previous argument there is then a function $f$ with $K \prec f \prec O$ and $\rho(f)>n$. Hence $\mu(O)=\sup \{\rho(f): f \prec O\}=\infty$. If $\mu(X)<\infty$, put $O=X$ in the previous argument. Then $\mu(X)=\sup \{\rho(f): f \prec X\}=\|\rho\|<\infty$. The proof is complete.

Theorem 3.9 (The representation theorem). Let $X$ be a locally compact Hausdorff space.

1. To each quasi-measure $\mu$ in $X$ there is a unique quasi-integral $\rho$ on $C_{c}(X)$ such that for any $f \in C_{c}(X)$ we have

$$
\rho(\phi(f))=\int \phi(i) d \mu_{f}
$$


for all $\phi \in\{\phi \in C(\operatorname{sp} f): \phi(0)=0\}$. Here $\mu_{f}$ is the regular Borel measure in $\boldsymbol{R}$ corresponding to $\mu$ and $f$.

2. Conversely, for any quasi-integral $\rho$ on $C_{c}(X)$ there is a unique quasi-measure $\mu$ in $X$ such that $\rho$ is the quasi-integral corresponding to $\mu$. Specifically we have, for any open set $O \subset X$ :

$$
\mu(O)=\sup \{\rho(f): f \prec O\} .
$$

Proof. The first part of the theorem follows from Proposition 3.7 Suppose $\rho$ is a quasi-integral on $C_{c}(X)$. Define a set function $\mu: \mathcal{O} \rightarrow \mathbf{R} \cup\{\infty\}$ by (3.1). Extend $\mu$ to the closed subsets $F$ of $X$ by $\mu(F)=\inf \{\mu(O): F \subset O, O$ is open $\}$. Notice that this implies $\mu(K)=\inf \{\rho(f): f \succ K\}$ when $K$ is compact by Urysohn's lemma and the monotonicity of $\rho$. We will show that $\mu$ is a quasi-measure in $X$. Note that $\mu(A)<\infty$ when $A \in \mathcal{A}^{*}$ by Urysohn's lemma and Corollary 3.5. Suppose that $O_{1}$ and $O_{2}$ are open disjoint subsets of $X$. Pick $f_{i}$ with $f_{i} \prec O_{i}$ and $\rho\left(f_{i}\right)>\mu\left(O_{i}\right)-\epsilon$ for $i=1,2$. We have $f_{1} f_{2}=0$ which implies $f_{1}, f_{2} \in \mathbf{A}\left(f_{1}-f_{2}\right)$ and thus

$$
\begin{aligned}
\mu\left(O_{1} \cup O_{2}\right) & \geq \rho\left(f_{1}+f_{2}\right)=\rho\left(f_{1}\right)+\rho\left(f_{2}\right) \\
& \geq \mu\left(O_{1}\right)+\mu\left(O_{2}\right)+2 \epsilon .
\end{aligned}
$$

Conversely if $f \prec O_{1} \cup O_{2}$, the opposite equality follows from observing that $f=$ $f_{1}+f_{2}$ where $f_{i}(x)=f(x)$ if $x \in O_{i}$ and elsewhere zero. Let $K \subset O \subset X$ where $K$ is compact and $O$ is open. By Urysohn's lemma there is an open bounded set $U$ and functions $f_{K}, f_{U}$ such that $K \subset U \subset \bar{U} \subset O, K \prec f_{K} \prec O$ and $\bar{U} \prec f_{U} \prec O$ with $\rho\left(f_{U}\right)>\mu(O)-\epsilon$. Then $f_{K}, f_{U} \in \mathbf{A}\left(f_{K}+f_{U}\right)$ and $f_{U}-f_{K} \prec O \backslash K$; thus

$$
\begin{aligned}
\mu(O \backslash K) & \geq \rho\left(f_{U}-f_{K}\right)=\rho\left(f_{U}\right)-\rho\left(f_{K}\right) \\
& >\mu(O)-\mu(K)-\epsilon,
\end{aligned}
$$

which yields $\mu(O) \leq \mu(O \backslash K)+\mu(K)$ when $\mu(O)<\infty$ and equality when $\mu(O)=\infty$. Conversely, if $f \prec O \backslash K$ with $\rho(f)>\mu(O \backslash K)-\epsilon$, then $K^{\prime}=\operatorname{supp} f \subset O \backslash K$, so $\left(X \backslash K^{\prime}\right) \cap U$ is an open set containing $K$. Pick $f_{K}$ such that $K \prec f_{K} \prec\left(X \backslash K^{\prime}\right) \cap U$; then $f f_{K}=0$. We have

$$
\begin{aligned}
\mu(O) & \geq \rho\left(f_{K}+f\right)=\rho\left(f_{K}\right)+\rho(f) \\
& >\mu(K)+\mu(O \backslash K)-\epsilon .
\end{aligned}
$$

We have shown that $\mu$ is a quasi-measure in $X$. The uniqueness of $\mu$ follows from Lemma 3.8. Let $\rho_{\mu}$ denote the quasi-integral corresponding to $\mu$; it remains to prove that $\rho_{\mu}$ is equal to $\rho$. Let $f \in C_{c}(X)$ be arbitrary. Then $\rho_{f}: \phi \rightarrow \rho(\phi(f))$ is a functional on $\{\phi: \phi \in C(\operatorname{sp} f), \phi(0)=0\}$. Extend $\rho_{f}$ to a functional $F$ on $C(\operatorname{sp} f)$ by $F(\phi)=\rho_{f}(\phi-\phi(0))+\phi(0), \phi \in C(\operatorname{sp} f)$. Now sp $f$ is compact and thus $F$ determines a unique regular Borel measure $\nu_{f}$ on $\operatorname{sp} f$ such that

$$
F(\phi)=\rho(\phi(f))=\int \phi(i) d \nu_{f} \text { when } \phi \in C(\operatorname{sp} f) \text { and } \phi(0)=0 .
$$

So by regularity it suffices to show that $\mu_{f}=\nu_{f}$ on the open subsets of $E=$ sp $f \backslash\{0\}$. Suppose $\epsilon>0$ and $U \subset E$ open are arbitrary. Pick a compact set $K \subset U$ such that $\nu_{f}(K)>\nu_{f}(U)-\epsilon$. Choose an Urysohn function $K \prec \phi \prec U$; then $\nu_{f}(U)-\epsilon<\nu_{f}(K) \leq \rho(\phi(f)) \leq \mu\left(f^{-1}(U)\right)=\mu_{f}(U)$ since $\phi \circ f \prec f^{-1}(U)$. Conversely, pick a compact set $K \subset f^{-1}(U)$ such that $\mu(K)>\mu\left(f^{-1}(U)\right)-\epsilon$ and a function $\phi$ with $f(K) \prec \phi \prec U$. Then since $\phi \circ f \succ f^{-1}(K)$ we have

$$
\mu_{f}(U)-\epsilon=\mu\left(f^{-1}(U)\right)-\epsilon<\mu(K) \leq \rho(\phi \circ f)=\rho_{f}(\phi) \leq \nu_{f}(U) .
$$

The proof is complete. 
Corollary 3.10. Let $\rho$ be a quasi-integral on $C_{c}(X)$. If $\rho$ is bounded, then $\rho$ has a unique extension to a quasi-integral on $C_{0}(X)$.

Proof. By Corollary [3.6 $\rho$ is uniformly continuous. Extend $\rho$ by continuity to a function $\rho_{0}: C_{0}(X) \rightarrow \mathbf{R}$. For example by the functions $\phi_{\epsilon}$ defined by

$$
\phi_{\epsilon}(x)= \begin{cases}0, & x<\epsilon, \\ 2 x-2 \epsilon, & \epsilon \leq x \leq 2 \epsilon, \\ x, & x>2 \epsilon .\end{cases}
$$

Obviously $\rho_{0}(\alpha f)=\alpha \rho_{0}(f)$ for all $\alpha \in \mathbf{R}, f \in C_{0}(X)$. Suppose $f \in C_{0}(X)$ and $\phi_{1}(f), \phi_{2}(f) \in \mathbf{A}_{0}(f)$. Then $\phi_{i}\left(\phi_{\epsilon}(f)\right) \in \mathbf{A}_{0}\left(\phi_{\epsilon}(f)\right)$ for all $\epsilon>0$ and $i=1,2$. Note that $\phi_{i}\left(\phi_{\epsilon}(f)\right)$ converges uniformly to $\phi_{i}(f)$ when $\epsilon$ tends to zero. Hence by continuity

$$
\begin{aligned}
\rho\left(\phi_{1}(f)+\phi_{2}(f)\right) & =\lim _{\epsilon \rightarrow 0} \rho\left(\phi_{1}\left(\phi_{\epsilon}(f)\right)+\phi_{2}\left(\phi_{\epsilon}(f)\right)\right) \\
& =\lim _{\epsilon \rightarrow 0}\left[\rho\left(\phi_{1}\left(\phi_{\epsilon}(f)\right)\right)+\rho\left(\phi_{2}\left(\phi_{\epsilon}(f)\right)\right)\right] \\
& =\rho\left(\phi_{1}(f)\right)+\rho\left(\phi_{2}(f)\right) .
\end{aligned}
$$

We have shown that $\rho_{0}$ is a quasi-integral on $C_{0}(X)$. The uniqueness of the extension is immediate from the continuity of $\rho_{0}$. The proof is complete.

\section{REFERENCES}

[1] J. F. Aarnes: "Quasi-states and Quasi-measures," Advances in Mathematics, vol. 86 (1991). MR 92d:46152

[2] J. F. Aarnes: "Image transformations and attractors," Dept. of Math., University of Trondheim, Preprint no. 2 (1994).

[3] J. F. Aarnes: "Quasi-measures in locally compact spaces," Norwegian University of Science and Technology, Preprint no. 1 (1996).

[4] J. F. Aarnes and A. B. Rustad: "Probability and Quasi-measures -a new interpretation," To appear in Math. Scand.

[5] W. Rudin: "Real and complex analysis," 3d ed., McGraw-Hill Book Company, Singapore (1987). MR 88k:00002

[6] A. B. Rustad: "The multidimensional median as a quasi-measure," Norwegian University of Science and Technology, Preprint no. 5 (1998).

[7] G. Taraldsen: "Image-transformations and quasi-measures in locally compact Hausdorff spaces," University of Trondheim, Preprint (1995).

Faculty of Mathematics, Norwegian University of Science and Technology, Sem Saelandsv 9, Gloshaugen, 7055 Dragvoll, Norway

E-mail address: alfr@math.ntnu.no

Current address: Department of Mathematical Sciences, Lade Norwegian University of Science and Technology, 7491 Trondheim, Norway 